\title{
Docência em tempos digitais: análise do perfil e da ação do professor frente às tecnologias em cenários escolares
}

\author{
Maristela Compagnoni Vieira ${ }^{1,2}$, Lucila Maria Costi Santarosa ${ }^{1}$, Débora \\ Conforto ${ }^{1,3}$ \\ ${ }^{1}$ PGIE, UFRGS - Porto Alegre - RS - Brazil. ${ }^{2}$ Centro Universitário Fadergs - Porto \\ Alegre - RS - Brazil. ${ }^{3}$ Rede Marista \\ maricompagnoni@gmail.com, lucilamcs@yahoo.com, \\ deboraconforto@gmail.com
}

\begin{abstract}
This study is based on TPACK and SAMR frameworks to analyze the influence of teacher and school identity elements on the composition of the technological pedagogical content knowledge profile and on the uses of educational technology (ET) among 606 teachers from 17 institutions. The main results indicate the existence of a relation between TPACK profile and age group $(p=0,001) ;$ SAMR performance and ET training $(p=0,002)$; and high incidence of teacher-centered practices. The research provides to the scientific and educational community extensions of the applied frameworks and new instruments with high consistency that allows the mapping of the teaching profile, the infrastructure and the school methodological context.
\end{abstract}

Resumo. Este estudo parte dos frameworks TPACK e SAMR para analisar a influência de elementos identitários docentes e escolares na composição do perfil de conhecimento tecnológico pedagógico do conteúdo e nos usos da tecnologia educacional (TE) entre 606 professores de 17 instituições. Os principais resultados indicam a existência de relação entre perfil TPACK e faixa etária ( $p=0,001)$; desempenho SAMR e capacitação em TE $(p=0,002) ;$ e a elevada incidência de práticas centradas no professor. A pesquisa entrega à comunidade científica e educacional ampliações dos frameworks aplicados e novos instrumentos com alta consistência que possibilitam o mapeamento do perfil docente, da infraestrutura e do contexto metodológico escolar.

\section{Introdução}

O ousado desafio de transformar os sistemas educacionais é um caminho que passa pela integração qualitativa das tecnologias digitais nos processos de ensino e de aprendizagem. No entanto, a tecnologia é apenas uma pequena peça de um quebracabeça amplo, que se conecta a outras partes fundamentais, como novas formas de compreender a representação do conhecimento, o ensino, a aprendizagem, o currículo, a avaliação e a formação dos professores [Costa 2013; Fagundes 2011; Valente 1999, 2013; Warschauer e Ames 2010; Warschauer 2011]. A contemporaneidade, em paralelo, nos proporcionou analisar cenários antes inimagináveis de computação ubíqua nas escolas, mas seu uso permanece, em muitos casos, servindo como veículos para metodologias tradicionais anacrônicas. 
Diante desse cenário, os frameworks TPACK e SAMR ${ }^{1}$ constituem-se como importantes ferramentas para repensar, compreender e contribuir para a mobilização de usos transformadores da tecnologia nos contextos educacionais, sobretudo no que diz respeito à formação dos professores para a utilização da tecnologia, da pedagogia e do conteúdo em atividades que proponham o protagonismo dos estudantes.

Este estudo emerge deste contexto, com o objetivo de analisar a influência de elementos identitários docentes e escolares na composição do perfil de conhecimento tecnológico e pedagógico do conteúdo de professores e nos usos da tecnologia educacional efetivados por eles em uma rede privada de ensino. Para levar a cabo este objetivo, fizeram-se necessárias criações e ampliações de instrumentos e ferramentas para realização da coleta e interpretação de dados relacionados aos professores e às instituições escolares nas quais atuavam, os quais foram analisados em suas possíveis relações e efeitos.

\section{Os frameworks TPACK e SAMR}

Koehler e Mishra (2009) propõem que uma abordagem de integração bem-sucedida da tecnologia na ação docente requer que os professores desenvolvam novas formas de compreender e acolher a complexidade afeita ao processo educativo: no centro de um bom ensino com a tecnologia residem três componentes principais: o conteúdo, a pedagogia e a tecnologia, além das relações que se estabelecem entre eles. Estas três bases de conhecimento formam o núcleo do framework TPACK (Figura 1), o qual foi construído a partir da teoria de Shulman (1986) acerca do PCK (Pedagogical Content Knwoledge, ou Conhecimento Pedagógico do Conteúdo).

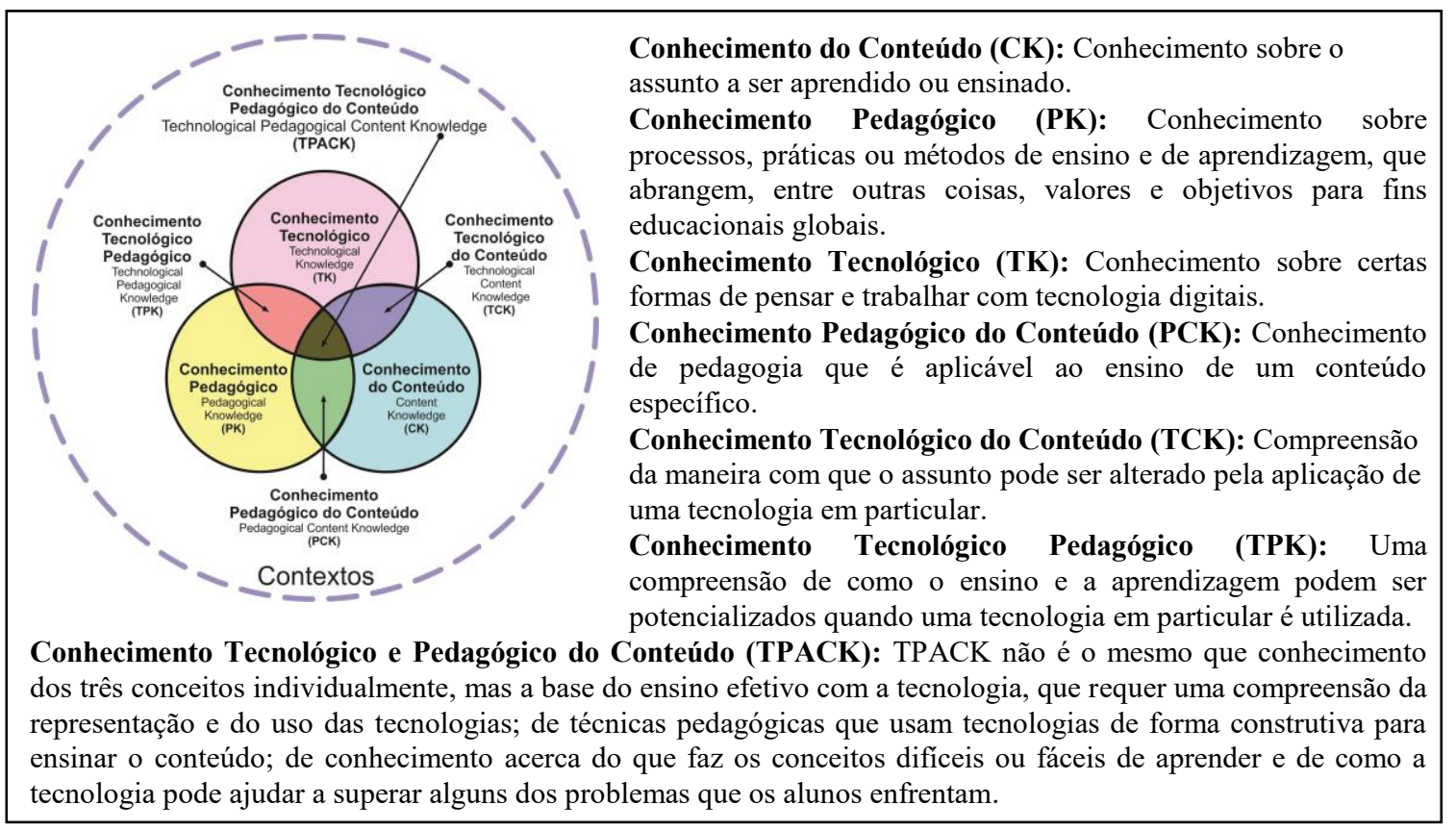

Figura 1. Conhecimento tecnológico e pedagógico do conteúdo (TPACK). Fonte: tpack.org (tradução nossa).

\footnotetext{
${ }^{1}$ Os acrônimos representam as definições em inglês para os frameworks "Technological Pedagogical Content Knowledge" e "Substitution Augmentation Modification Redefinition", respectivamente.
} 
VII Congresso Brasileiro de Informática na Educação (CBIE 2018)

Anais dos Workshops do VII Congresso Brasileiro de Informática na Educação (WCBIE 2018)

Para Mishra e Koehler (2006), a tecnologia é tipicamente vista como um "conjunto separado de conhecimentos e habilidades que não precisam ser aprendidos", e o relacionamento entre essas habilidades e as "habilidades testadas e verdadeiras (conteúdo e pedagogia)" (p. 1025) também não são reconhecidas ou são consideradas muito triviais para serem adquiridas ou implementadas. Como consequência dessa perspectiva, os programas de formação de professores são com frequência projetados com foco na aprendizagem acerca de um equipamento ou software específicos, com atenção insuficiente a sua relação com o conteúdo e a pedagogia.

O framework SAMR (Figura 2) configura-se como um instrumento para auxiliar e avaliar a maneira como professores incorporam tecnologias em suas aulas, de forma a possibilitar maior compreensão acerca de seus usos pedagógicos, bem como daquelas que apresentam maiores ou menores efeitos na aprendizagem dos estudantes [Puentedura, 2008]. Estrutura-se, hierarquicamente, em duas camadas, denominadas Melhora e Transformação, nas quais se inserem quatro níveis de exploração da mediação tecnológica. As camadas representadas pelos termos Melhora e Transformação indicam que, enquanto no primeiro, a utilização das tecnologias envolve apenas uma melhora das ações pedagógicas, no segundo ocorre transformação dos processos pedagógicos, com implicações mais significativas na aprendizagem dos alunos.

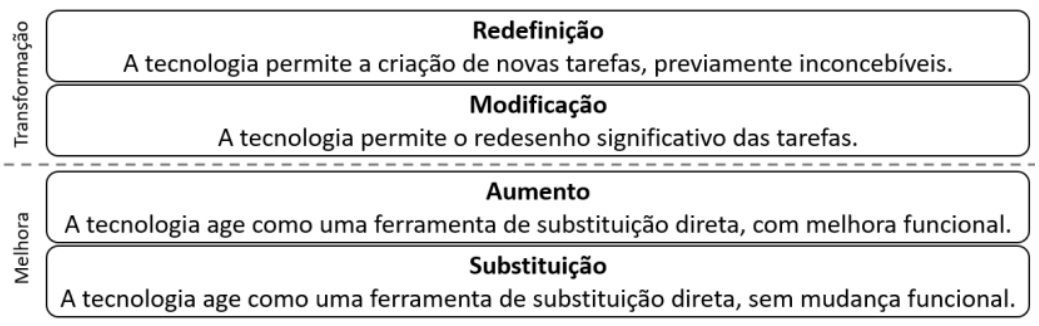

Figura 2. Framework SAMR. Fonte: As autoras.

\section{Métodos}

A coleta de dados ocorreu por meio da adaptação e da ampliação do instrumento proposto por Schmidt et al. (2009). Obteve-se, ao final do processo, um questionário composto por quatro conjuntos de questões: (1) perguntas demográficas, (2) perguntas em relação ao conhecimento tecnológico e pedagógico do conteúdo com respostas do tipo likert, (3) perguntas em relação ao contexto metodológico da instituição na qual a professor atua e que podem ser considerados facilitadores ou entreves da integração da tecnologia em propostas pedagógicas e (4) perguntas dissertativas nas quais o professor descreve práticas docentes nas quais ocorreram, na sua percepção, a integração de tecnologias, pedagogia e conteúdo. A população do estudo foi composta por 606 professores que atuam nos Ensinos Fundamental e Médio de 17 escolas privadas de Educação Básica que integram uma rede nacional de ensino.

A análise estatística envolveu a aplicação dos seguintes recursos: teste de normalidade de Shapiro-Wilk, análise de variância (ANOVA) one-way ou teste de Kruskal-Wallis, Modelo de Equações de Estimações Generalizadas (GEE), teste de comparação múltipla de Bonferroni, correlação de Pearson ou de Spearman e Alfa de Cronbach. Relativamente à técnica utilizada para análise dos dados obtidos em questões 
VII Congresso Brasileiro de Informática na Educação (CBIE 2018)

Anais dos Workshops do VII Congresso Brasileiro de Informática na Educação (WCBIE 2018)

abertas, optou-se pela análise textual discursiva, conforme proposta de Moraes e Galiazzi (2013).

Embora tenha-se partido da ferramenta de Schmidt et al. (2009), o instrumento ${ }^{2}$ utilizado neste estudo é fruto de traduções, adaptações e ampliações que objetivaram aproximar a ferramenta do contexto nacional e ampliar o escopo de profissionais a que se destina, além de incluir elementos que abarcaram recursos conceituais advindos das teorias de Becker (2000) e Cuban (2013), oferecendo ainda ferramentas para a análise dos itens discursivos por meio da ampliação do framework SAMR. A análise estatística demonstrou que o perfil TPACK apresentou relação significativa com o desempenho SAMR, ou seja, que escores mais altos no perfil TPACK estão relacionados a escores mais altos em SAMR, assim como o inverso $(\mathrm{p}<0,001)$, dado que corrobora a validade da utilização do framework SAMR para a interpretação das questões discursivas.

O instrumento elaborado ampliou o foco da análise dos anos iniciais para toda a Educação Básica, além de incluir dimensões destinadas a revelar a percepção docente quanto a (1) elementos como estruturações espaçotemporais alternativas à sala de aula tradicional organizada em fileiras, (2) aprendizagem problematizadora e centrada no aluno, (3) diferenciação no uso de tecnologias de uso geral e específicas, tecnologia assistiva, (4) exploração de recursos na perspectiva BYOD (Bring Your Own Device) e (5) quanto à presença de um profissional mediador para o uso das tecnologias digitais na escola. Além disso, no decorrer do processo de atualização do instrumento, foi adicionada uma dimensão externa ao perfil docente a fim de compreender os elementos percebidos pelos professores como entraves metodológicos ou estruturais à utilização da TE. Com base nos aspectos discutidos por Cuban (2013), estruturou-se um conjunto de sete afirmações em escala likert, seguidas de uma questão aberta para a descrição de situações que dificultam a integração da tecnologia em aula.

A ampliação do instrumento para professores de outros níveis de ensino exigiu a definição de um conjunto de conteúdos específicos das disciplinas de cada área do conhecimento. Esse processo envolveu quatro etapas: (1) análise do documento Base Nacional Comum [Brasil 2017] e da Matriz Curricular da rede pesquisada; (2) estruturação do conjunto de conteúdos globais de cada disciplina, com base nos documentos consultados; (3) validação dos conteúdos sumarizados pelos especialistas da rede e (4) formulação da versão final do conjunto de conteúdos globais de cada disciplina.

No que tange à aferição da consistência ou confiabilidade do instrumento desenvolvido, utilizou-se o Coeficiente Alpha $(\alpha)$ de Cronbach, tendo-se observado alta confiabilidade para todas as dimensões da ferramenta, conforme Tabela 1. Para Mateo (2012), correlações situadas entre o intervalo 0,8 e 1 podem ser consideradas muito altas, enquanto Nunnally (1978) indica que um instrumento ou teste é classificado como tendo fiabilidade apropriada quando o $\alpha$ é pelo menos 0,70 .

\footnotetext{
${ }^{2}$ A versão final digital pode ser acessada no formato enviado aos professores, em que a apresentação das seções depende das respostas do usuário (http://bit.ly/instrumentotpack) e em formato sequencial e linear, que permite visualizar todas as questões e seções sem necessidade de preenchimento para direcionamento a áreas específicas (http://bit.ly/intrumentolinear).
} 
VII Congresso Brasileiro de Informática na Educação (CBIE 2018)

Anais dos Workshops do VII Congresso Brasileiro de Informática na Educação (WCBIE 2018)

Tabela 1: Alpha ( $\alpha$ ) de Cronbach geral de cada dimensão do instrumento

\begin{tabular}{c|c}
\hline Dimensão & Alpha $(\alpha)$ de Cronbach geral \\
\hline Contexto Metodológico e Tecnológico & 0,825 \\
Conhecimento Tecnológico & 0,884 \\
Conhecimento Tecnológico Pedagógico & 0,854 \\
Conhecimento Pedagógico & 0,907 \\
Conhecimento do Conteúdo & 0,955 \\
Conhecimento Pedagógico do Conteúdo & 0,944 \\
Conhecimento Tecnológico do Conteúdo & 0,900 \\
Conhecimento Tecnológico Pedagógico do Conteúdo & 0,926 \\
\hline
\end{tabular}

Embora estudos anteriores também tenham alcançado alta confiabilidade para o instrumento TPACK original [Shmidt et al. 2009; Almera, Diaz e Garrido 2015], a condução de nova análise se fez fundamental para garantir que as ampliações e adaptações realizadas em todas as dimensões de análise mantiveram a fiabilidade da ferramenta. Nesse sentido, pode-se afirmar, diante dos resultados obtidos para $\alpha$, que o instrumento proposto não apenas se manteve confiável, como os $\alpha$ relacionados a algumas dimensões mostraram-se até mesmo superiores aos resultados alcançados para o instrumento original [Shmidt 2009; Almera, Diaz e Garrido 2015].

\section{Descrição e Discussão dos Resultados}

Para responder à questão relativa a quais elementos identitários docentes e escolares estão relacionados na composição do perfil de conhecimento tecnológico e pedagógico do conteúdo de professores e os reflexos desse perfil nos usos de tecnologia educacional em uma rede privada de ensino de âmbito nacional, foram elaboradas hipóteses de pesquisa a partir das quais serão apresentados e discutidos, a seguir, os resultados.

\section{Quadro 1. Hipóteses de pesquisa.}

\begin{tabular}{|c|l|}
\hline Hipótese & \multicolumn{1}{c|}{ Descrição } \\
\hline 1 & $\begin{array}{l}\text { Elementos demográficos como (a) idade, (b) nível de formação, (c) capacitação na área das } \\
\text { tecnologias educacionais e (d) área de atuação têm relação com os resultados do (e) perfil TPACK e } \\
\text { (f) desempenho SAMR. }\end{array}$ \\
\hline 2 & Há relação entre o perfil TPACK e o desempenho SAMR. \\
\hline 3 & $\begin{array}{l}\text { Há relação entre o perfil TPACK e o desempenho SAMR e o (g) parque tecnológico da instituição } \\
\text { na qual os professores atuam. }\end{array}$ \\
\hline 4 & $\begin{array}{l}\text { Há relação entre o perfil TPACK e o desempenho SAMR e a percepção quanto aos } \\
\text { (h) contextos metodológicos, infraestruturais e tecnológicos da instituição na qual os professores } \\
\text { atuam. }\end{array}$ \\
\hline 5 & $\begin{array}{l}\text { Há relação entre o tipo de dispositivo (tablets, notebooks, lousas digitais, computadores de mesa) e } \\
\text { as configurações espaçotemporais de utilização (laboratórios de informática, laboratórios móveis, } \\
\text { salas de aula, makerspaces) na forma como os professores propõem atividades pedagógicas } \\
\text { envolvendo o uso de tecnologias digitais. }\end{array}$ \\
\hline
\end{tabular}

No que tange à hipótese 1 , a análise das relações entre faixa etária e o perfil TPACK revelou diferença entre as médias do perfil TPACK e os grupos etários ( $\mathrm{p}=$ 0,001). Observou-se desempenho superior para o grupo na faixa etária compreendida entre os 27 e os 38 anos. No entanto, na análise do comportamento do perfil TPACK dos grupos etários em relação a cada uma das dimensões, percebe-se que esses só apresentam distanciamento superior ao dos demais nas dimensões relacionadas à tecnologia. A Figura 3 descreve o desempenho de cada grupo etário em relação às dimensões TPACK, individualmente. 


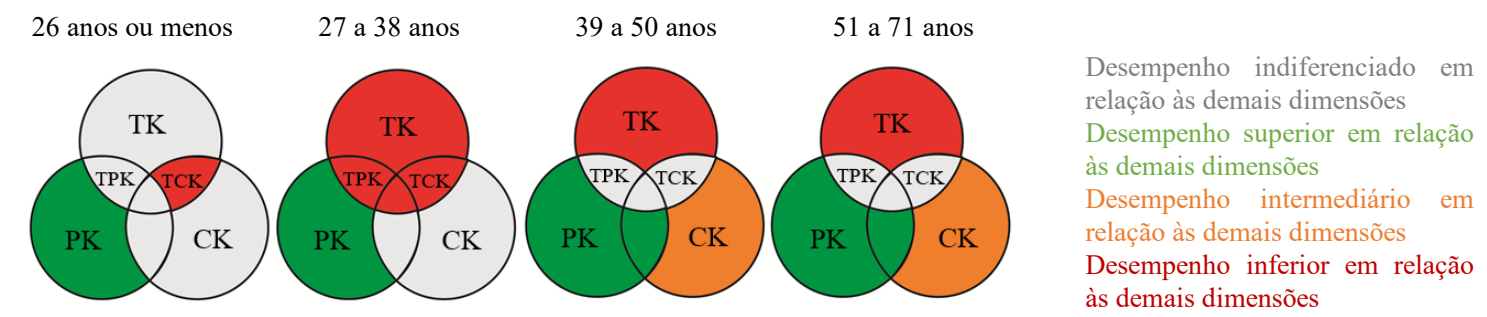

Figura 3. Desempenho individual dos grupos etários em relação às dimensões

O nível de formação dos professores demonstrou impactar o desempenho SAMR $(\mathrm{p}=0,048)$, com escores mais baixos para indivíduos com formação média ou Graduação, e mais elevados para Mestres - vale aqui ressaltar que o grupo de Doutores pode ter sido numericamente insuficiente para inferência de relações mais significativas. Não houve, no entanto, mudanças no comportamento do perfil TPACK em relação à variável nível de formação $(p=0,392)$.

A variável de capacitação na área das tecnologias educacionais demonstrou-se a mais elucidativa em termos de relação com o desempenho SAMR $(p=0,002)$. Professores que relataram ter experiência em capacitações consistentes na área das tecnologias educacionais, apresentaram desempenho significativamente superior para SAMR do que aqueles sem nenhuma capacitação na área. É interessante observar também que o desempenho daqueles professores que descreveram ter vivenciado capacitações breves ou esparsas, como treinamentos de um ou dois turnos isolados, não se diferenciou do comportamento dos demais, indicando pouca validade para ações de capacitação de curta duração, descontextualizadas.

Outra consideração ainda é pertinente quanto à influência da variável de formação na área da tecnologia educacional e o desempenho SAMR. Professores que descrevem formações mais amplas na área frequentemente participaram dessas formações por conta própria, o que pode indicar interesse ou motivação individuais que se refletem, posteriormente, em uma maior aplicação prática de tais conhecimentos, enquanto que os professores que descrevem formações mais breves em geral as fizeram no ambiente profissional, como um imperativo ou necessidade colocados pela instituição a qual estão vinculados.

Quanto às áreas de atuação, observou-se, tanto em TPACK $(\mathrm{p}=0,002)$ quanto em SAMR ( $<<0,001)$, relações positivas mais significativas com os professores de Ciências Humanas que, na análise qualitativa, descreveram usos pedagógicos da tecnologia mais abertos e relacionados à criação. Esses resultados contrariam a percepção da gestão de tecnologia da rede, que percebia os professores de Matemática como aqueles que mais exploravam tecnologias em suas aulas. Os professores de Matemática, no entanto, foram aqueles que mais descreveram apropriação pedagógica de aplicativos e softwares específicos da sua área de atuação, como Geogebra e Poly.

A hipótese 2, que mostrou-se significativa para a relação TPACK e SAMR ( $p<$ 0,001) implicou no desenvolvimento de estratégias de mensuração dos itens discursivos, com base em SAMR. Partindo da análise dos pressupostos relacionados às pedagogias e propostas centradas no professor e no aluno (Figura 4), elaborou-se um eixo complementar para interpretação do framework SAMR, que identifica as camadas de 
VII Congresso Brasileiro de Informática na Educação (CBIE 2018)

Anais dos Workshops do VII Congresso Brasileiro de Informática na Educação (WCBIE 2018)

melhora e transformação como mais centradas no aluno ou no professor. Com a atualização do framework SAMR pela inclusão desse foco de análise, somaram-se aos níveis originalmente propostos, substituição, aumento, modificação e redefinição, oito tipos de propostas: consumação, reprodução, edição, comunicação, criação, colaboração, circulação e difusão.

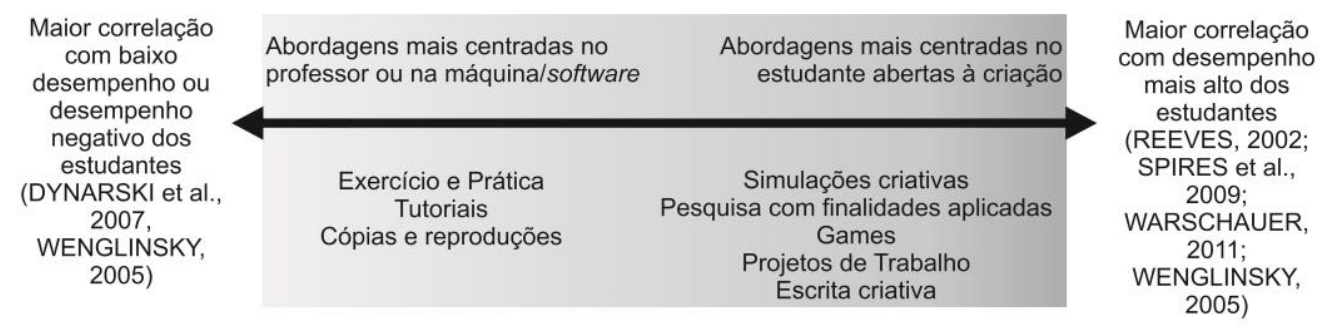

Figura 4. Distribuição das propostas de uso pedagógico da tecnologia
conforme correlação com desempenho dos estudantes.

A análise dos relatos dos professores em relação ao framework SAMR (Figura 5) indica que há uma significativa proporção de propostas classificadas no nível de melhora e, notadamente, na camada de aumento. A ampliação do framework permite as observar que a maior concentração de atividades descritas pelos professores encontra-se na camada de aumento.

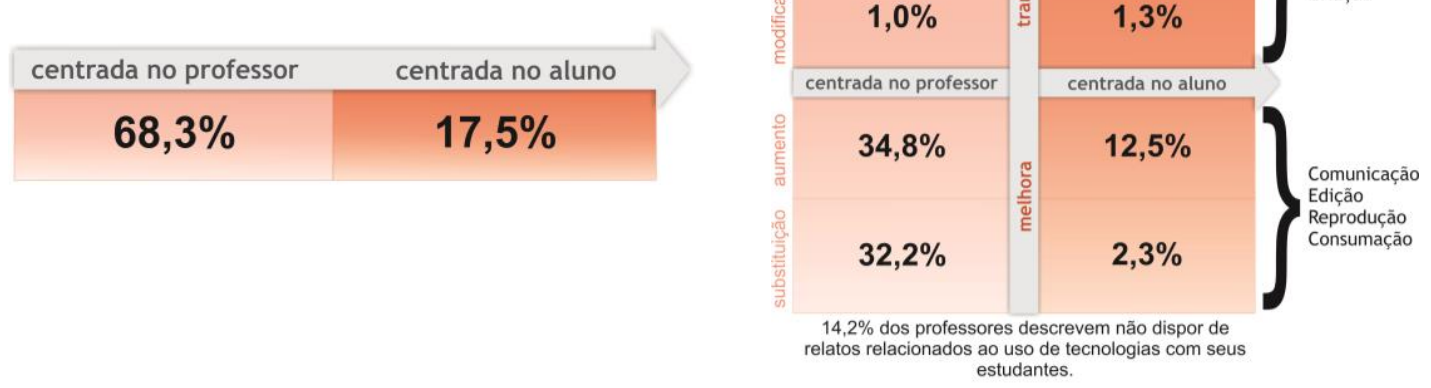

Figura 5. Análise dos discursos em relação aos níveis e camadas SAMR

Em relação à hipótese 3, os resultados deste estudo não demonstram correlação entre o parque tecnológico ${ }^{3}$ das escolas e o perfil TPACK ou o desempenho SAMR, o que, frente à análise de outros resultados, possibilita a percepção de que sejam mais significativos para a qualificação da utilização da tecnologia nas escolas o investimento (a) em processos de discussão e estudo que levem a mudanças, sobretudo, em concepções pedagógicas, promovendo ações educativas mais centradas no estudante e em metodologias ativas; (b) na construção de políticas institucionais voltadas ao uso

\footnotetext{
${ }^{3}$ Dente os instrumentos desenvolvidos e validados na condução deste estudo está um modelo matemático para avaliação do parque tecnológico de instituições educativas. Elencou-se os principais tipos de distribuição e utilização da tecnologia educativa, e para cada um deles, atribuiu-se um peso, com base nas possibilidades de desenvolvimento pedagógico identificadas no referencial teórico que embasa o estudo. Informações completas do modelo estão descritos na tese da qual deriva este artigo.
} 
ético e qualificado das TE, sobretudo no que tange ao acesso à internet e às políticas de BYOD; (c) no fortalecimento de profissionais docentes que desempenhem lideranças institucionais, como fomentadores do uso pedagógico da tecnologia junto aos professores de áreas específicas, na promoção de ações interdisciplinares e criativas que explorem diferentes espaços e recursos e (d) em um espaço institucional de formação docente consistente e de longo prazo, voltado ao fortalecimento do perfil TPACK e do desempenho SAMR com ações centradas no estudante e na criação e no qual sejam capitalizadas as ações internas de sucesso entre pares docentes.

Relativamente à hipótese 4 , a análise da percepção dos professores quanto ao contexto metodológico e tecnológico - derivada das contribuições de Cuban (2013), quanto às razões pelas quais os professores não utilizam as tecnologias disponíveis nas escolas - apresentou relação com a média de todas as dimensões TPACK (p 0,035).

A verificação da hipótese 5 aponta para a existência de relação entre o tipo de dispositivo ou as configurações espaçotemporais de utilização e a forma como os professores propõem atividades pedagógicas. $\mathrm{O}$ universo pesquisado neste estudo indica que tecnologias como lousa digital interativa, tablet e celular apresentam uma incidência maior de usos centrados no professor do que os laboratórios de informática, robótica e makerspace.

Os usos relacionados às lousas indicaram que esses recursos cumpriram papel de "digitalizadores" de processos historicamente relacionados à aula expositiva. Enquanto que o reduzido poder de processamento, de armazenamento e de interações por meio da escrita em dispositivos móveis remetem seus usos a atividades de consumo.

A maior incidência de atividades centradas no professor ocorreu em tecnologias exploradas na sala de aula, um espaço historicamente centrado na figura do docente, com configurações espaciais que, com frequência, favorecem a organização da rotina de estudos na forma de palestras. Outros ambientes, como os laboratórios de informática, robótica e os recentes makerspaces, que se organizam espacialmente de formas em geral diversas, podem influenciar na descentralização da ação pedagógica, aproximando-se mais da figura do estudante. Além disso, em geral, ambientes desenhados para exploração de recursos tecnológicos dentro da escola (como os laboratórios de informática ou de robótica), contam com a intervenção de um profissional específico, com expertise na utilização dos recursos disponíveis e que podem compensar eventuais dificuldades do professor em relação ao conhecimento tecnológico, na lógica TPACK.

Em que pesem as características dos espaços e dos equipamentos, a utilização de ambientes como os laboratórios de informática e makerspace requeria agendamento prévio. Contrariamente, as lousas digitais, mais presentes nas escolas envolvidas, oferecem um panorama mais amplo do uso que grande parte dos professores (e não apenas aqueles mais inclinados a usos construtivos ou colaborativos) faz da tecnologia.

\section{Considerações Finais}

Para além dos resultados evidenciados nas respostas às hipóteses elaboradas para a condução deste estudo, a pesquisa demandou o desenvolvimento de instrumentos, modelos e frameworks que, tendo sido necessários para a coleta de dados, hoje se configuram como produtos entregues à comunidade científica e educacional relacionada 
à área da Informática na Educação: (1) Instrumento, com alta consistência interna, para identificação do perfil tecnológico e pedagógico do conteúdo para professores em serviço na Educação Básica, com adequação às recentes atualizações nacionais no que diz respeito aos conteúdos curriculares propostos pela Base Nacional Comum. Sua replicação pode ser facilmente realizada, de forma completa ou segmentada por áreas de atuação; (2) Instrumento para análise da percepção docente quanto aos elementos que dificultam ou potencializam a utilização da tecnologia em contextos metodológicos e infraestruturas escolares; (3) Ampliação do framework SAMR, que, ao dividir verticalmente os diferentes níveis e camadas originalmente propostos, aplica uma conceituação mais detalhada acerca da utilização de tecnologias em contextos pedagógicos, direcionando a percepção para as práticas que evidenciam maior protagonismo e criação por parte dos estudantes; (4) Correlações significativas entre os resultados do perfil TPACK e o desempenho inferido pelo framework SAMR ampliado, indicando que os dois frameworks podem ser complementarmente utilizados nos processos de compreensão da percepção e da ação docente frente à integração de tecnologias em atividades de ensino e de aprendizagem; (5) Modelo matemático para análise do parque tecnológico de instituições escolares fundamentado em referencial teórico e em concepções pedagógicas que contextualizam as tecnologias educacionais na perspectiva de empoderamento dos estudantes e dos professores para a criação de possibilidades de aprendizagem centradas seu protagonismo e na potencialização de recursos móveis e de espaços de criação.

O conjunto de análises derivadas deste estudo corrobora a discussão atual acerca da invalidez da tecnologia em conduzir, por si, à modificação ou à qualificação dos processos educativos. Embora seja uma ferramenta que contenha, em si, grande potencial, a ausência de um processo intencional de discussão e análise da pedagogia , que, ao fim, deve ser o fio que conduz sua exploração, permanecerá produzindo pouco ou nenhum resultado para a transformação da escola e para a formação das crianças e jovens ou qualificação dos seus processos de ensino e de aprendizagem. Muito mais do que o verniz que encobre práticas anacrônicas, a tecnologia deve ser compreendida e agenciada como uma das peças que compõem um cenário educativo mais amplo para o qual pode contribuir e no qual pedagogia e professores devem estar sob maior destaque.

\section{Referências}

Almenara, J.C.; Díaz, Y.M.; Garrido, C.C. (2015) Validación de la aplicación del modelo TPACK para la formación de lo professorado em TIC. Revista d'Inovación Educativa, n. 14, jan./jun..

Becker, H.J. (2015) Findings from the Teaching, Learning and Computing Survey: Is Larry Cuban Right? Educacional Policy Analysis Archives, v.8, n.51, 2000. Disponível em: http://www.crito.uci.edu/tlc/findings/ccsso.pdf. Acesso em: 10 jul.

Brasil. Ministério da Educação. (2017). Base Nacional Comum Curricular. Terceira versão. Brasília: MEC. Disponível em: http://bit.ly/2mHJEhf. Acesso em: jul. 2018.

Costa, F.A. (2013) O potencial transformador das TIC e a formação de professores e educadores. In: Almeida, M.E.B de A.; Dias, P.; Silva, B.D. da (Orgs). Cenários de Inovação para a educação na sociedade digital. São Paulo: Edições Loyola, p. 47-74. 
VII Congresso Brasileiro de Informática na Educação (CBIE 2018)

Anais dos Workshops do VII Congresso Brasileiro de Informática na Educação (WCBIE 2018)

Cuban, L. (2013) Why so many structural changes in schools and so little reform in teaching practice? Journal of Educational Administration, Vol. 51 Iss 2, pp.109 - 125.

Koehler, M. J., \& Mishra, P. (2009) What is technological pedagogical content knowledge? Contemporary Issues in Technol. and Teacher Education, 9(1), p.60-70.

Mateo, J. (2012) La investigación expost-facto. In: BISQUERRA, R. (coord.) Metodología de investigación educativa. Madrid: La Muralla, p. 195-229.

Mishra, P,; Koehler, M. (2006) Technological Pedagogical Content Knowledge: A new framework for teacher knowledge. Teachers College Record, 108(6), pp.1017-1054.

Moraes, R; Galiazzi, M. do C. (2013) Análise textual discursiva. Ijuí: Editora Unijuí.

Nunnally, J. C. (1978) Psychometric theory. New York: McGraw-Hill Inc.

Puentedura, R. (2008) Models for enhancing tecnhnology integration (excerpeted from Rubem R. Puentedura TPCK and SAMR). Disponível em $<$ http://www.msad54.org/sahs/TechInteg/mlti/SAMR.pdf >. Acesso em 28/12/2016.

Schmidt, D.; Baran, E.; Thompson, A.; Mishra, P.; Koehler, M.; Shin, T. (2009) Technological Pedagogycal Content Knowledge (TPACK): The development and validation of an assessment instrument for preservice teachers. Journal of Research on Technology on Education, volume 42, número 2, pp 123-149.

Shulman, L. (1986) Those who understand: Knowledge growth in teaching. Educational Researcher, 15 (2), 4-14.

Valente, J. A. As tecnologias e as verdadeiras inovações na educação. (1999). In: Valente, J.A. Informática na Educação no Brasil: análise e contextualização histórica. Campinas: UNICAMP/NIED.

Warschauer, M. (2011) Learning in the cloud: How (and Why) to Transform Schools with Digital Media. New York: Teachers College Press.

Warschauer, M.; Ames, M. (2010) Can one laptop per child save the world's poor? Journal of International Affairs, v. 64, n. 1.

Dynarski, M.; Agodini, R.; Heaviside, S.; Novak, T.; Carey, N.; Campuzano, L. et al. Effectiveness of reading and mathematics software products: Findings from the first student cohort. Washington DC: Department of Education, 2007.

Reeves, D. Accountability in action. Denver: Advanced Learning Press, 2002.

Spires, H.A.; Wiebe, E.; Young, C.A.; Hollebrands, K.; Lee, J.K. Toward a new learning ecology: teaching and learning in 1:1 enviroments. Raleigh, NC: Friday Institute for Educational Innovation, North Carolina State University, 2009.

Wenglinsky, H. Using Technology Wisely: The Keys to success in schools. Nova Iorque: Teachers College Press, 2005 